\title{
HLA and KIR genotyping correlates with relapse after T-cell-replete haploidentical transplantation in chronic myeloid leukaemia patients
}

\author{
X-Y Zhao ${ }^{1}$, Y-J Chang ${ }^{1}$, L-P Xu ${ }^{1}$, X-H Zhang ${ }^{1}$, K-Y Liu ${ }^{1}$, D Li ${ }^{1}$ and X-J Huang ${ }^{\star}, 1,2$ \\ ${ }^{1}$ Peking University People's Hospital and Peking University Institute of Hematology, Beijing Key Laboratory of Hematopoietic \\ Stem Cell Transplantation, Beijing 100044, China and ${ }^{2}$ Peking-Tsinghua Center for Life Sciences, Beijing 100871, China
}

Background: Conflicting results have been reported regarding the predicative roles of alloreactive natural killer (NK) cells on the outcomes of transplantation in leukaemia patients.

Methods: We prospectively analysed the human leukocyte antigen (HLA) typing of donor-recipient pairs and the KIR typing of the donors in $97 \mathrm{CML}$ patients to address the predictive roles of NK cells in relapse undergoing T-cell-replete haploidentical transplantation.

Results: Patients with class I ligands for the donor-inhibitory KIR gene exhibited decreased molecular and haematologic relapse rates $(P=0.003$ and $P=0.015$, respectively). There was a significantly reduced risk of molecular and haematologic relapse in patients with HLA-C1C2 or C2C2 who accepted donors with KIR2DS1 or in patients with HLA-Bw4 who accepted donors with KIR3DS1 ('recipient with relevant KIR ligand for donor-activating KIR', $n=25)$, compared with the remaining transplants $(n=72$, $P=0.009$ and $P=0.009$, respectively). In addition, the presence of class I ligand in the recipients of donor-activating KIR contributed to a decreased relapse rate in patients lacking class I ligand in the recipient of donor-inhibitory KIR $(P=0.04$ and $P=0.03$, respectively).

Conclusions: This study suggests that the presence of class I ligands for the donor-activating or donor-inhibitory KIR gene in the recipient might confer some protection against leukaemic relapse in T-cell-replete haploidentical transplantation.

Natural killer (NK) cells are lymphocytes that are critical for innate immunity against malignant or virally infected cells. In recipients, the functions of NK cells are primarily modulated by the expression of activating and inhibitory receptors, as well as their relevant ligands (Raulet and Vance, 2006). The alloreactivity of NK cells depends on the expression of a KIR and its MHC-I ligand in the donor (for education) and on the absence of the MHC-I ligand in the recipient (for relief from inhibition). In biological studies of NK cells, two general models that define NK alloreactivity have arisen: a mismatch between the donor and recipient killer immunoglobulin receptor (KIR) ligands (ligand-ligand model); or recipients lacking KIR ligands for donor-inhibitory KIR receptors (missing self-model) (Ruggeri et al, 2002; Kim et al, 2005). Furthermore, in alloreactive NK-cell responses, KIR2DS1 expression offers a remarkable advantage because it allows for the efficient killing of $\mathrm{C} 2 / \mathrm{C} 2$ or $\mathrm{C} 1 / \mathrm{C} 2$ myelomonocytic dendritic cells (DCs) and T-cell blasts (Sivori et al, 2011). The interaction between KIR3DS1 and human leukocyte antigen (HLA)-Bw4 was predictive of progression-free survival after autologous stem cell transplantation (SCT) in patients with multiple myeloma (Gabriel et al, 2010). Thus, KIR2DS1 and KIR3DS1 expression in HSC donors could substantially increase the size of the alloreactive NK-cell subset. 
Family HLA-mismatched/haploidentical haematopoietic SCT is a feasible therapeutic approach for patients with lethal malignant haematological diseases who lack an HLA-matched donor. Several haploidentical transplant protocols have been established worldwide (Huang, 2008; Fuchs et al, 2010). Human leukocyte antigen mismatches can trigger donor vs recipient NK-cell alloreactivity (Ruggeri et al, 1999, 2007; Leung et al, 2004, 2005; Zhao et al, 2011). However, conflicting results have been reported regarding the impact of the ligand-ligand model or the missing self-model on the outcomes of transplantation in leukaemic patients (Zhao et al, 2011). More interestingly, in the setting of T-cell-replete haploidentical HSCT, we observed that a KIR ligand mismatch was a strong risk factor for a high incidence of relapse and decreased overall survival (OS), in direct contrast to the findings of Perugia's group regarding T-cell depletion (Huang et al, 2007). Moreover, in another limited series of T-cell-replete haploidentical transplants, recipients lacking KIR ligands for donor-inhibitory KIR receptors showed a tendency towards a higher relapse rate (Zhao et al, 2007). Although these prior results have been inconsistent or even contradictory, the reasons for this discordancy are currently unclear, but they might reflect differences in patient heterogeneity, the involvement of donor-activated KIR genes and post-transplantation alloreactive NK-cell reconstitution.

CML is considered to be one of the leukaemias most susceptible to immune manipulation (Simula et al, 2007). Yong et al (2009) demonstrated that in vitro, primitive, quiescent CD34 + cells in CML were targeted by expanded NK cells. Kijima et al (2011) demonstrated that missing self-recognition mediated by NK cells protected mice from primary CML in vivo, suggesting that NK-cell recognition had the potential to cure CML. Our previous report showed that HLA-mismatched/haploidentical HSCT was a feasible therapeutic option for CML patients without an HLA-matched sibling (Xiao-jun et al, 2008; Jiang et al, 2011). Considering that the conflicting roles of NK cells in protection against leukaemia, inferred from retrospective haploidentical transplantation analysis, might have been caused by patient heterogeneity, we prospectively analysed the HLA typing of donor-recipient pairs and the KIR typing of the donor in relatively purified samples from patients with CML, to address further the predictive roles of NK cells in the relapses of patients undergoing T-cell-replete haploidentical transplantation. These transplants are particularly important to investigate, because they allow for the evaluation of the influence of inhibitory and activating donor KIR types in the presence in recipients of the relevant KIR ligands on clinical outcomes without the confounding effects of disease heterogeneity. The findings could lead to better donor selection between donors and recipients.

\section{PATIENTS AND METHODS}

Patients. We enrolled patients with CML who were suitable for allo-HSCT and who had no related or unrelated HLA-identical donors. In total, 134 patients with CML underwent T-cell-replete HLA-haploidentical transplantation between August 2003 and December 2009 at the Peking University Institute of Hematology. Written informed consent was obtained from all of the patients and donors before their entry into the study, in accordance with the Declaration of Helsinki. In this cohort, 37 patient-donor pairs refused to undergo KIR and HLA-C genotyping. Therefore, the remaining 97 consecutive CML patients were enrolled in the study. Both the patients and their donors provided written informed consent, and the Institutional Review Board of the Peking University Institute of Hematology approved this study. The characteristics of the 97 patients are presented in Table 1.

Conditioning regimen, mobilisation and collection of stem cells, and GVHD prophylaxis. All of the patients received myeloablative regimens, including a combination of cytosine arabinoside (Ara-C) $\left(4 \mathrm{~g} \mathrm{~m}^{-2} \times 2\right.$ days, on days -10 and -9$)$, busulfan $\left(12 \mathrm{mg} \mathrm{kg}^{-1}\right.$ administered in 12 doses over 3 days, on days $-8,-7$ and -6$)$, cyclophosphamide $\left(1.8 \mathrm{~g} \mathrm{~m}^{-2} \times 2\right.$ days, on days -5 and -4$)$, Simustine (MeCCNU) $\left(250 \mathrm{mg} \mathrm{kg}^{-1}\right.$ on day -3), and rabbit anti-human thymocyte immunoglobulin (thymoglobulin $2.5 \mathrm{mg} \mathrm{kg}^{-1}$ per day i.v. of the Sangstat product) on days -5 through -2 .

The donors received rhG-CSF (Filgrastim) $5 \mu \mathrm{g} \mathrm{kg}^{-1}$ daily for 5-6 days. On the fourth day, bone marrow (BM) cells were harvested. The target total nucleated cell count was $3.0 \times 10^{8}$ (median, $3.77 \times 10^{8}$, range, $0.24-8.16 \times 10^{8}$ ) cells per $\mathrm{kg}$ of recipient weight. On the fifth and sixth days, peripheral blood progenitor cells (PBPCs) were collected. The target mononuclear cell count was $3.0 \times 10^{8}$ (median, $3.58 \times 10^{8}$, range, $0.91-11.7 \times 10^{8}$ ) cells per $\mathrm{kg}$ of recipient weight. The fresh and unmanipulated BM and PBPCs were infused into the recipients on the day of collection.

The prophylaxis for GVHD included cyclosporine A (CsA) and short-term methotrexate (MTX) with mycophenolate mofetil (MMF) treatment. Cyclosporine A was started intravenously on day -9 at a dose of $2.5 \mathrm{mg} \mathrm{kg}^{-1}$ and was switched to the oral formulation as soon as the patient was able to tolerate oral medication after engraftment. The dosage was adjusted to the patients' blood levels. Mycophenolate mofetil was administered orally at a dose of $0.5 \mathrm{~g}$ every $12 \mathrm{~h}$, from day -9 before transplantation to day 30 after transplantation and then $0.25 \mathrm{~g}$ every $12 \mathrm{~h}$ for $1-2$ months. The dosage of MTX was $15 \mathrm{mg} \mathrm{m}^{-2}$, and it was administered i.v. on day 1 , while the dose was $10 \mathrm{mg} \mathrm{m}^{-2}$ on days 3, 6 and 11 after transplantation. The diagnosis and grading of GVHD were established according to published criteria. In all of the recipients, rhG-CSF was administered subcutaneously at a dose of $5 \mu \mathrm{g} \mathrm{kg}^{-1}$ per day from day 6 after transplantation until the neutrophil count reached $0.5 \times 10^{9}$ cells per litre for 3 consecutive days (Xiao-jun et al, 2008; Jiang et al, 2011).

Outcome definition. The haematologic response criteria were defined as follows. Complete haematologic remission (CHR) was defined as a myeloblast count of $\leqslant 5 \%$ in the BM, no myeloblasts in the peripheral blood, neutrophil and platelet counts of at least $1.5 \times 10^{9}$ per litre and $100 \times 10^{9}$ per litre, respectively, and no evidence of extramedullary involvement. Complete molecular remission (CMR) was defined as an undetectable BCR-ABL transcript level. Post-transplantation molecular relapse was defined as positive BCR-ABL transcripts confirmed in two consecutive assays after previously achieving CMR or a persistent BCR-ABL transcript increase of more than $1 \mathrm{log}$. Cytogenetic relapse was defined as Philadelphia $(\mathrm{Ph})$ chromosome reappearance, and haematologic relapse was defined according to the criteria of the World Health Organization (WHO). Event-free survival was defined as the time that elapsed between allo-HSCT and the appearance of one of the following events: the absence of haematologic response at 3 months; the loss of previously obtained CHR, MCR or CCR; post-transplantation molecular relapse; relapse in AP or BP; or death from any cause. Overall survival was defined as the time from the beginning of allo-HSCT to death from any cause (Xiao-jun et al, 2008; Jiang et al, 2011).

Minimal residual disease monitoring. Minimal residual disease (MRD) was evaluated in recipient BM cells on days 30,60, 90, 120, 150 and 180 and then every 1 year after HSCT. Cytogenetic analysis was performed by fluorescence in situ hybridisation (FISH). BCR-ABL transcripts were detected by analysing BM with nested reverse transcriptase PCR before 2004 and with quantitative real-time PCR after 2004. The normalisation ratios of BCR-ABL transcript levels in quantitative PCR analysis were obtained through comparison with the levels of $\mathrm{ABL}$ transcript, as 


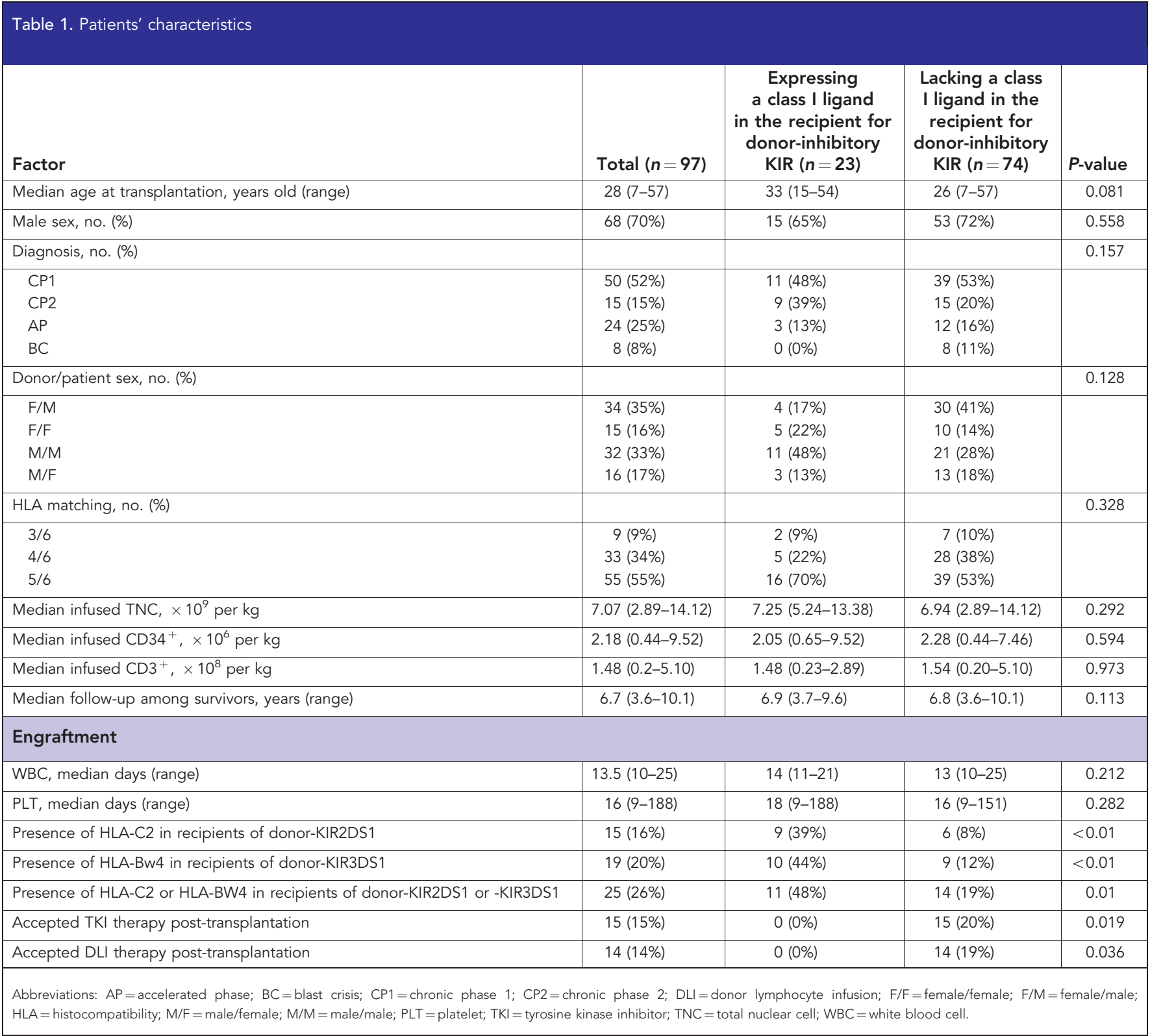

previously reported (Xiao-jun et al, 2008; Jiang et al, 2011). The sensitivity of the Bcr-Abl assay for detecting MRD was $0.001 \%$.

Imatinib and donor lymphocyte infusion. Donor lymphocyte infusion (DLI) and imatinib mesylate were given to patients who had a molecular, cytogenetic or haematologic relapse after HSCT, following a trial of immunosuppressant withdrawal. In patients without GVHD, G-CSF-mobilised peripheral blood stem cell grafts were used for DLI. Otherwise, imatinib mesylate was given. The initial dose of imatinib mesylate was $400 \mathrm{mg}$ per day, and it was adjusted, when necessary, according to response and tolerance (Xiao-jun et al, 2008; Jiang et al, 2011).

HLA and KIR genotyping. Patient and donor DNA was prepared from peripheral blood mononuclear cells and was used for HLA typing and KIR genotyping before transplantation. The samples were stored at $-40^{\circ} \mathrm{C}$. Serological and molecular HLA typing were performed as described by Lu et al. Recipient HLA-C and HLA-B alleles were identified by high-resolution DNA-based HLA typing and were segregated, where appropriate, into the epitope groups HLA-C group 1 (HLA-C ${ }^{\text {Asn80 }}$ : HLA-Cw1, 3, 7, 8, 13, 14 alleles), HLA-C group 2 (HLA-C ${ }^{\text {Lys80 }}$ : HLA-Cw2, 4, 5, 6, 12, 15, 17, 18 alleles), and HLA-Bw4.
The KIR genotyping was performed on genomic DNA from haploidentical donors of haematopoietic stem cells, using PCR amplification with sequence-specific primers, as described previously, or using a KIR genotyping kit (One Lambda, Canoga Park, CA, USA), according to the manufacturer's instructions (Huang et al, 2007; Zhao et al, 2007).

Definitions of two models of NK-cell alloreaction. The following KIR receptors and their ligands were considered: KIR2DL1 with HLA-C 'group 2'-related alleles, KIR2DL2/3 with HLA-C 'group 1 '-related alleles, and KIR3DL1 with the HLA-Bw4 allele. The ligand-ligand model was defined as follows: patients were separated into two groups according to the HLA typing of the donors and patients, specifically those with or without a KIR ligand mismatch (the absence of one donor KIR ligand in the recipient) (Ruggeri et al, 2007). The missing self model was defined as follows: the missing-ligand model included all of the donor-recipient pairs in which there was a mismatch between at least one KIR inhibitory gene in the donor and the HLA molecule(s) in the recipient (Leung et al, 2004, 2005).

Statistical analysis. The associations between patient characteristics or pre-transplantation variables and post-transplantation 
outcomes were analysed using the Kaplan-Meier method, or they were calculated using cumulative incidence curves to accommodate competing risks. R software (R Development Core Team (2006). $\mathrm{R}: \mathrm{A}$ Language and Environment for Statistical Computing. $\mathrm{R}$ Foundation for Statistical Computing, Vienna, Austria. ISBN 3-900051-07-0, URL: http://www.R-project.org.) was used to calculate the cumulative incidence, considering the presence of competing risk. Event-free survival and OS were estimated using Kaplan-Meier methods, and the probabilities of relapse, transplant-related mortality (TRM), grade II to IV aGVHD, and both absolute neutrophil count (ANC) and platelet count (PLT) recovery were calculated as cumulative incidences to adjust the analysis for competing risks. To evaluate the independent effects of missing ligand transplants and KIR ligand-mismatched transplants, Cox regression analysis was performed. Factors included in the models were recipient and donor age, sex, sex mismatch, diagnosis (myeloid leukaemia vs lymphoid leukaemia), number of HLA-mismatched alleles, missing self transplants, KIR ligandmismatched transplants, pre-transplantation risk category (AP + $\mathrm{BC}+\mathrm{CP} 2$ vs $\mathrm{CP} 1$ ), and recipients with the relevant KIR ligand for donor-activated KIR. The final multivariate models were constructed using a forward stepwise model selection approach. The patient characteristics among the three groups were compared using chi-square statistics for categorical variables and with the Mann-Whitney $U$ test for continuous variables. $P<0.05$ was considered as statistically significant.

\section{RESULTS}

Patient characteristics. The patients' characteristics are provided in Table 1. In total, 96 of the 97 patients achieved WBC engraftment, and 92 of the 97 patients achieved PLT engraftment. One patient died on day 24 without WBC or PLT engraftment. All of the engrafted patients achieved complete donor chimaerism after transplantation. Sixty-five patients survived without leukaemia. Sixteen patients died of transplant-related complications after HSCT. Twenty-two patients had molecular relapse, of whom six accepted TKI therapy and returned to MMR, while the remaining sixteen patients progressed to haematological relapse. Eight of the sixteen relapsing patients died. In all, 2 patients did not complete the study, and 14 of 16 patients received DLI combined with TKI or chemotherapy. Of these 14 patients, 2 returned to MMR, and 6 patients are currently living with leukaemia. The median follow-up duration for the cohort was 6.7 years (range, 3.6-9.3 years). Of the 97 patients, there were 61 and 36 patients who had 0-I aGVHD and II-IV aGVHD, respectively. Among the 92 patients evaluated for the cGVHD who survived longer than 100 days, 58 patients had limited (31 patients) or extensive (27 patients) cGVHD.
The frequencies of inhibitory and activating KIR genes in this Chinese population were consistent with the gene frequencies reported in previously published studies. Specifically, the frequencies were $98 \%$ for KIR2DL1, 28\% for KIR2DL2, 100\% for KIR2DL3 $100 \%$, 93\% for KIR3DL1, 32\% for KIR2DS1, $11 \%$ for KIR2DS2, $15 \%$ for KIR2DS3, 29\% for KIR2DS5, 32\% for KIR3DS1, and 55\% for KIR haplotype BX.

Presence of a class I ligand in the recipient for donor-inhibitory KIR contributed to a decreased relapse rate. The data obtained as of 1 August 2013 were analysed, and the univariate effects of the demographic data on post-transplantation outcomes were analysed by the Kaplan-Meier method or were calculated using cumulative incidence curves to accommodate for competing risks.

There were 74 donor-recipient pairs lacking a class I ligand in the recipient for donor-inhibitory KIR and 23 present a class I ligand in the recipient for donor-inhibitory KIR. As shown in Figure 1, univariate analysis demonstrated that there were significantly increased molecular and haematologic relapse rates in patients lacking a class I ligand in the recipient for donorinhibitory KIR (group A, $n=74$ ), compared with those expressing a class I ligand in the recipient for donor-inhibitory KIR (group B, $n=23, P=0.003$ and $P=0.015$, respectively, Figure $1 \mathrm{~A}$ and $\mathrm{B}$ ). Therefore, patients lacking a class I ligand in the recipient for donor-inhibitory KIR had longer EFS ( $P=0.024$, Figure $1 C)$. There were similar incidences of aGVHD, cGVHD and TRM in patients lacking the KIR ligand for donor-inhibitory KIR, compared with those among patients expressing the KIR ligand for donorinhibitory KIR. The OS in patients expressing the KIR ligand for donor-inhibitory KIR tended to be longer than that of patients lacking the KIR ligand for donor-inhibitory KIR, although these differences were not significant $(P=0.137)$.

No significant influences of a KIR ligand mismatch between the donor and the recipient on clinical outcomes, including relapse $(P=0.722), \quad$ aGVHD $(P=0.916), \quad$ cGVHD $(P=0.838)$, TRM $(P=0.727)$, OS $(P=0.816)$ and $\operatorname{EFS}(P=0.656)$, were found in CML patients.

Presence of a class I ligand in the recipient for donor-activating KIR contributed to a decreased relapse rate. The activating donor KIR genes, including KIR2DS1, KIR2DS2, KIR2DS3, KIR2DS5, and KIR3DS1, were found to have no significant influences on relapse. KIR2DS1 expression represents a remarkable advantage because it allows for the efficient killing of $\mathrm{C} 2 / \mathrm{C} 2$ or $\mathrm{C} 1 / \mathrm{C} 2$ myelomonocytic DCs and T-cell blasts. In addition, in patients with multiple myeloma, the interaction between KIR3DS1 and HLA-Bw4 predicts progression-free survival after autologous SCT. We hypothesised that this association would be observed only in donors with KIR2DS1 or KIR3DS1 and recipients with HLA-C1C2 or $-\mathrm{C} 2 / \mathrm{C} 2$ or $\mathrm{Bw} 4$ because of the presence of a relevant KIR ligand for the activating KIR gene. We found a significantly reduced risk of molecular relapse among CML patients with HLA-C1C2 or -C2/C2
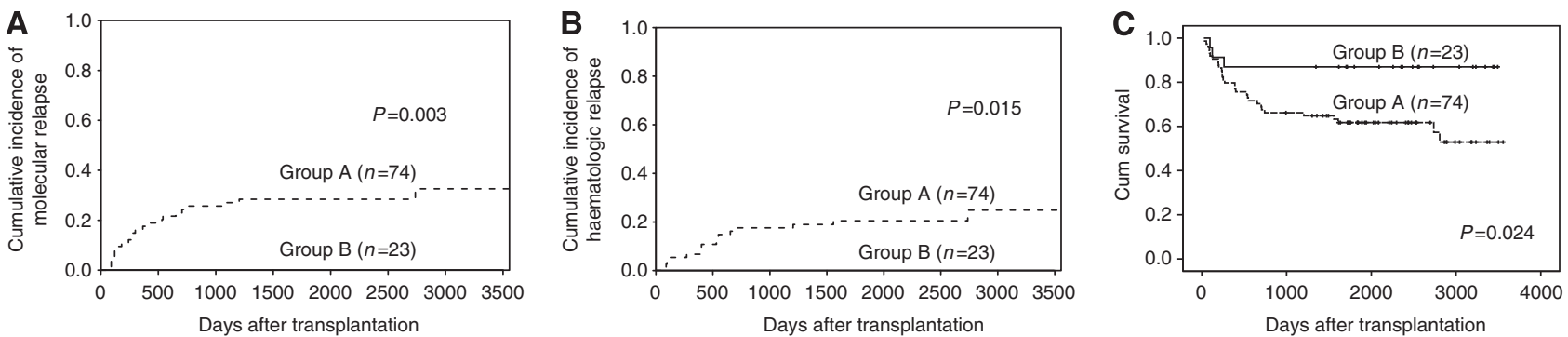

Figure 1. The effects of missing self between donors and recipients on clinical outcomes. The cumulative incidence estimates for the probability of molecular (A) and haematologic (B) relapse, as well as the Kaplan-Meier estimate for event-free survival (EFS, C) for CML patients lacking or expressing of the relevant KIR ligand (HLA-BW4 or HLA-C2 positive) for the donor-inhibitory KIR gene (group $A, n=74$ or group $B, n=23$ ) after haploidentical T-cell-replete transplantation. 
whose donors were positive for KIR2DS1, compared with patients with HLA-C1C1 whose donors were positive for KIR2DS1 $(P=0.008)$ or negative for KIR2DS1 $(P=0.024$, Figure 2A). Furthermore, the haematologic relapse rate among CML patients with $\mathrm{HLA}-\mathrm{C} 1 \mathrm{C} 2$ or $-\mathrm{C} 2 / \mathrm{C} 2$ whose donors were positive for KIR2DS1 was significantly lower than that of patients with HLA$\mathrm{C} 1 \mathrm{C} 1$ whose donors were positive for KIR2DS1 $(P=0.010$, Figure 2B).

Similar to the predictive roles of donor KIR2DS1 and recipient HLA-C ligand, patients with HLA-Bw4-accepting donors with KIR3DS1 positivity tended to have lower molecular and haematologic relapse rates, compared with patients with HLA-Bw4accepting donors with KIR3DS1 negativity $(P=0.058$ and $P=0.081$, respectively, Figure $3 \mathrm{~A}$ and $\mathrm{B})$. At the same time, molecular relapse in patients with HLA-Bw4-positive-accepting donors with KIR3DS1 positivity was significantly less common than in patients who were HLA-Bw4 negative $(P=0.048$, Figure 3A).

No effects of the recipients' HLA-Bw4 positivity or negativity on relapse were found. However, patients with HLA-C1C2 or -C2C2 $(n=59)$ had lower molecular relapse rate $(10.53 \pm 5.06 \%$ vs $34.72 \pm 8.01 \%, \quad P=0.026)$ and haematologic relapse rates $(8.08 \pm 4.55 \%$ vs $20.39 \pm 5.31 \%, P=0.066)$, compared with those of patients with HLA-C1C1 $(n=38)$.

Next, we combined the above predictive model, specifically subgrouping patients with HLA-C1C2- or C2C2-accepting donors with KIR2DS1-positive patients or patients with HLA-Bw4accepting donors with KIR3DS1 positive ('recipient with relevant KIR ligand for donor-activating KIR', $n=25$ ) and 'other' (the remaining transplants, $n=72$ ). The molecular relapse and haematologic relapse rates in patients in the 'recipient with relevant KIR ligand for donor-activating KIR' group were significantly lower than those of the patients in the 'other' group $(P=0.009$ and $P=0.009$, Figure $4 \mathrm{~A}$ and $\mathrm{B})$. The donor-activated KIR gene with the relevant KIR ligand in recipients, including aGVHD, cGVHD, TRM, OS and EFS, was found to have no significant influences on the clinical outcomes of CML patients.

Presence of class I ligand in the recipient for donor-activated KIR contributed to a decreased relapse rate in recipient lacking a class I ligand for donor-inhibitory KIR. Because patients lacking a class I ligand for donor-inhibitory KIR had higher relapse rates than those expressing a class I ligand for donor-inhibitory KIR, we examined whether activating donor KIR2DS1 positivity or KIR3DS1 positivity with relevant HLA ligand in recipients could overcome the higher relapse rate in patients with a missing self between the donor and the recipient. Among the 74 patients without the KIR ligand for the donor-inhibitory KIR gene, there were 13 patients in the 'recipient with relevant KIR ligand for donor-activating KIR' group and 61 patients in the 'other' group. The molecular relapse and haematologic relapse rates in patients in the 'recipient with relevant KIR ligand for donor-activating KIR' group were significantly higher than those of the patients in the 'other' group $(P=0.04$ and $P=0.03$, Figure 5$)$ among the patients with a missing self between the donor and the recipient.

There were no significant differences in recipient age, diagnosis, pre-transplantation risk category, HLA incompatibility, or doses of $\mathrm{CD} 3+\mathrm{T}$ cells and CD34 + cells among the patients with or without a missing self between the donor and the recipient (Table 2). However, there were more patients with the relevant KIR ligand for a donor-activated KIR gene in the 'recipient expressing a class I ligand for donor-inhibitory KIR' group, compared with
A

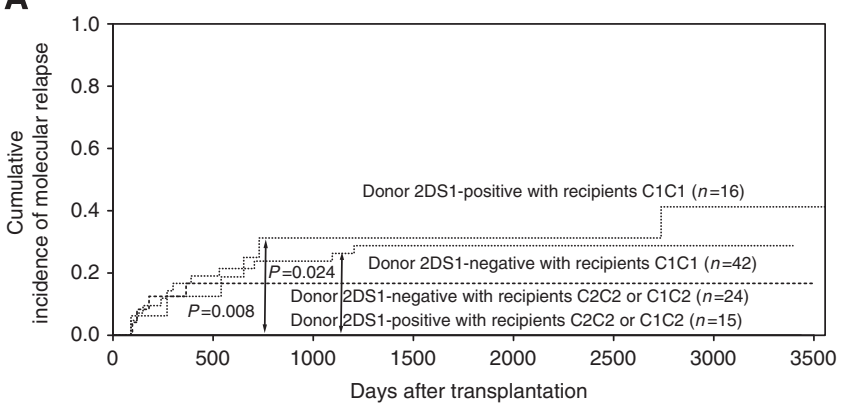

B

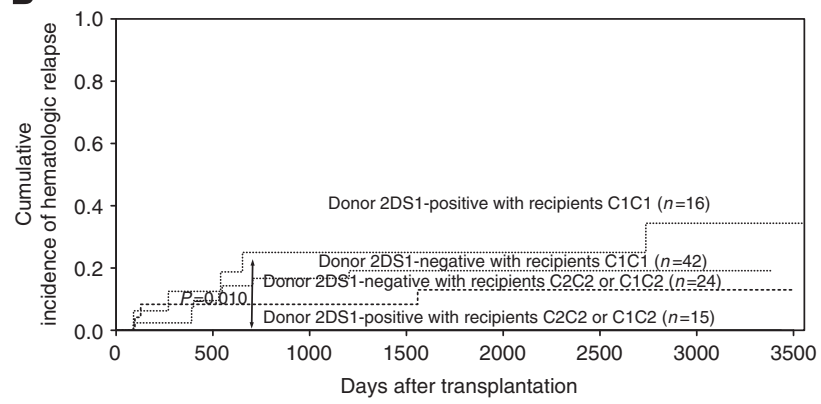

Figure 2. The effects of donor KIR2DS1 positivity on the relapse of recipients with HLA-C2 ligand. The cumulative incidence estimate for the probability of molecular (A) and haematologic (B) relapse in CML patients who were HLA-C2 positive (HLA-C1C2 or HLA-C2C2) accepting donors with KIR2DS1 positivity, compared with those patients who were HLA-C2 negative (HLA-C1C1) accepting donors with KIR2DS1 positivity or negativity after haploidentical T-cell-replete transplantation.
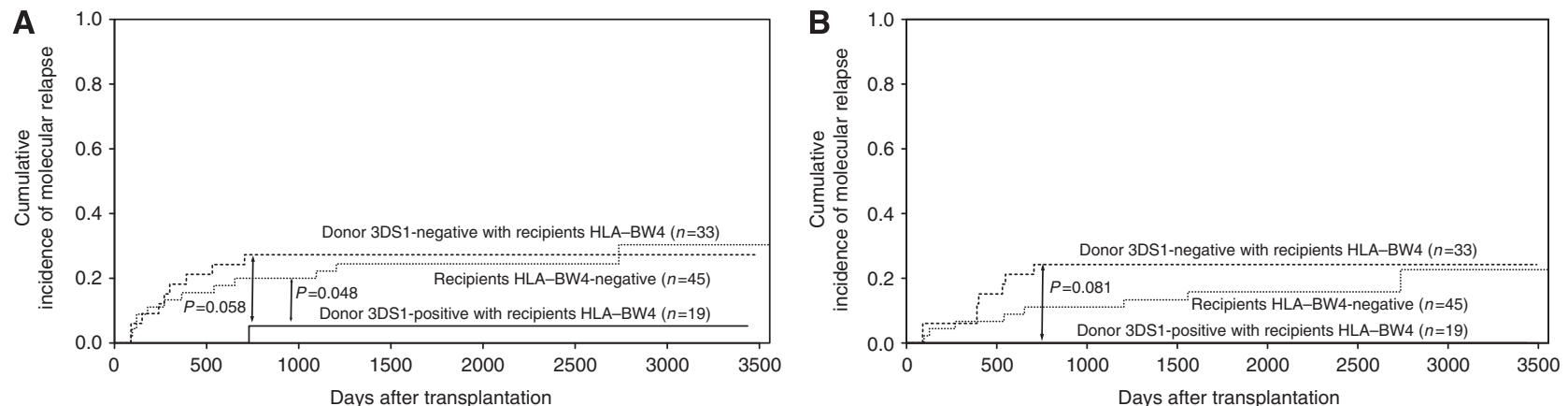

Figure 3. The effect of donor KIR3DS1 positivity on the relapse of recipients with HLA-Bw4 ligand. The cumulative incidence estimate for the probability of molecular (A) and haematological (B) relapse in CML patients with HLA-BW4 positive (HLA-BW4Bw4 or HLA-BW4BW6) accepting donors with KIR3DS1 positivity, compared with those patients with HLA-BW4 positive (HLA-BW4Bw4 or HLA-BW4BW6) accepting donors with KIR3DS1 positivity or those patients who were HLA-BW4 negative (HLA-BW6Bw6) after haploidentical T-cell-replete transplantation. 
A

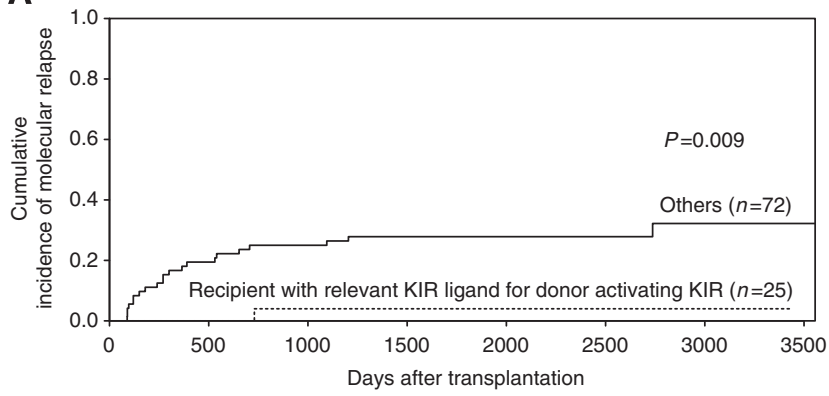

B

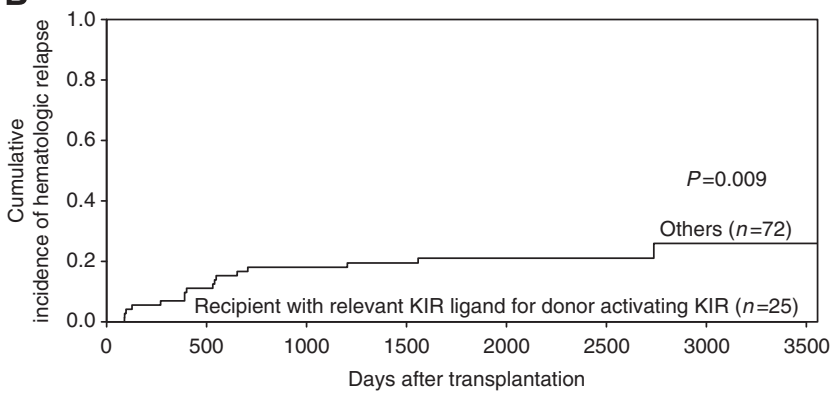

Figure 4. The effects of donor-activating KIR gene on the relapse of recipients with the relevant KIR ligand. The cumulative incidence estimate for the probability of molecular (A) and haematologic (B) relapse in CML patients with the relevant KIR ligand (HLA-BW4 or HLA-C2 positive) for donor-activating KIR gene (KIR2DS1 or KIR3DS1) after haploidentical T-cell-replete transplantation.

A

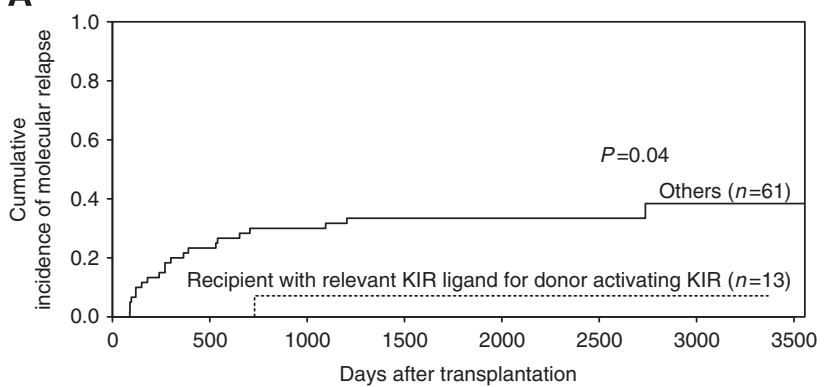

B

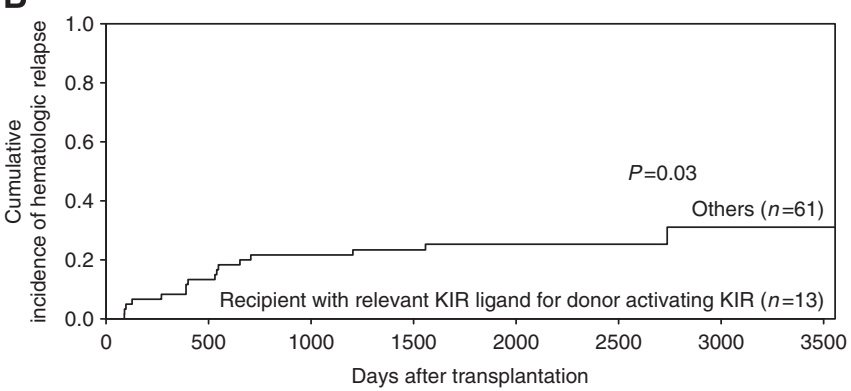

Figure 5. The effects of donor-activating KIR gene on the relapse of recipients with the relevant KIR ligand for the activating KIR gene but not for the inhibitory KIR gene. The cumulative incidence estimate for the probability of molecular (A) and haematologic (B) relapse in CML patients with the relevant KIR ligand (HLA-BW4 or HLA-C2 positive) for the donor-activating KIR gene (KIR2DS1 or KIR3DS1) but not for the donorinhibitory KIR gene (with missing self between donor and patient) after haploidentical T-cell-replete transplantation.

those in the 'recipient lacking a class I ligand for donor-inhibitory KIR' group (Table 2).

Multivariate analysis confirmed that 7-year molecular relapse was best prevented by the combination of recipients having a relevant KIR ligand (HLA-C2 positive or HLA-Bw4 positive) for accepting donors with activating KIR gene positivity (KIR2DS1 or KIR3DS1) (HR 0.141 (0.019-1.047)), $P=0.056$, Table 2). The absence of the KIR ligand for the donor-inhibitory KIR gene was also an independent risk factor for lower EFS (HR 3.102 (0.93710.266), $P=0.064$, Table 2). Additionally, multivariate analysis also demonstrated that high-risk CML $(\mathrm{CP} 2+\mathrm{AP}+\mathrm{BC})$ was an independent predictor of OS, EFS and relapse. Transplant-related mortality was encouraged by donor KIR2DS3 positivity (HR 4.290 (1.544-11.921), $P=0.005)$ and was prevented by donor KIR2DS5 positivity (HR $0.121(0.016-0.918), P=0.041)$. Therefore, donor KIR2DS3 and donor KIR2DS5 played contrasting roles in predicting OS and EFS post-transplantation (Table 2). At the same time, having a donor with the KIR BX haplotype was an independent risk factor for 3-4 aGVHD post-transplantation (HR 4.096 (1.175-14.275), $P=0.010)$.

\section{DISCUSSION}

In the current study, we investigated the predictive roles of distinct models for NK-cell alloreactivity on haploidentical transplantation outcomes in patients with CML. By limiting the transplant setting of our study to patients with Bcr-Abl-positive CML who received T-cell-replete haploidentical transplant, we avoided procedural heterogeneities, such as variabilities in the conditioning regimens or the GVHD prophylaxis used, as well as the disease heterogeneities, as observed in many other studies. To our surprise, although we avoided the impact of heterogeneous disease, we nevertheless demonstrated that, in this setting of T-cell-replete haploidentical HCT, CML patients lacking the ligand for their donors' inhibitory KIR tended to have increased relapse and inferior survival. Specifically, both the molecular and haematologic relapse rates were increased in recipient lacking a class I ligand for donor-inhibitory KIR. Therefore, the impact of therapeutic factors, such as DLI and TKI therapy for molecular relapse, was excluded. Furthermore, the patients' pre-transplantation CML risk levels were equal between the groups, and there were more patients receiving DLI or TKI therapy post-transplantation in the group of recipient lacking a class I ligand for donor-inhibitory KIR. However, this difference did not change the high relapse rate and inferior EFS in this group. Thus, the impact of disease heterogeneity on the conflicting effects of a missing self model was excluded. We found that a missing self model between donors and recipients was indeed associated with a high relapse rate in the setting of T-cell-replete transplantation, in agreement with our previous retrospective report (Huang et al, 2007; Zhao et al, 2007) and contrary to the findings reported in the setting of a potent T-cell-depleted haploidentical HSCT (Leung et al, 2004, 2005). Fuchs and colleagues also failed to find any significant differences in any patient outcomes when utilising the other proposed models, including ligand incompatibility, missing ligands, and gene-gene for stimulatory KIR, in the setting of non-myeloablative haploidentical HSCT study, utilising T-cell-replete grafts and a novel post-transplantation immunosuppressive regimen, including $\mathrm{Cy}$ (Symons et al, 2010). Furthermore, even in the setting of T-celldepleted haploidentical transplantation, Ruggeri et al (2007) did not find any beneficial effects of a missing self model on clinical 


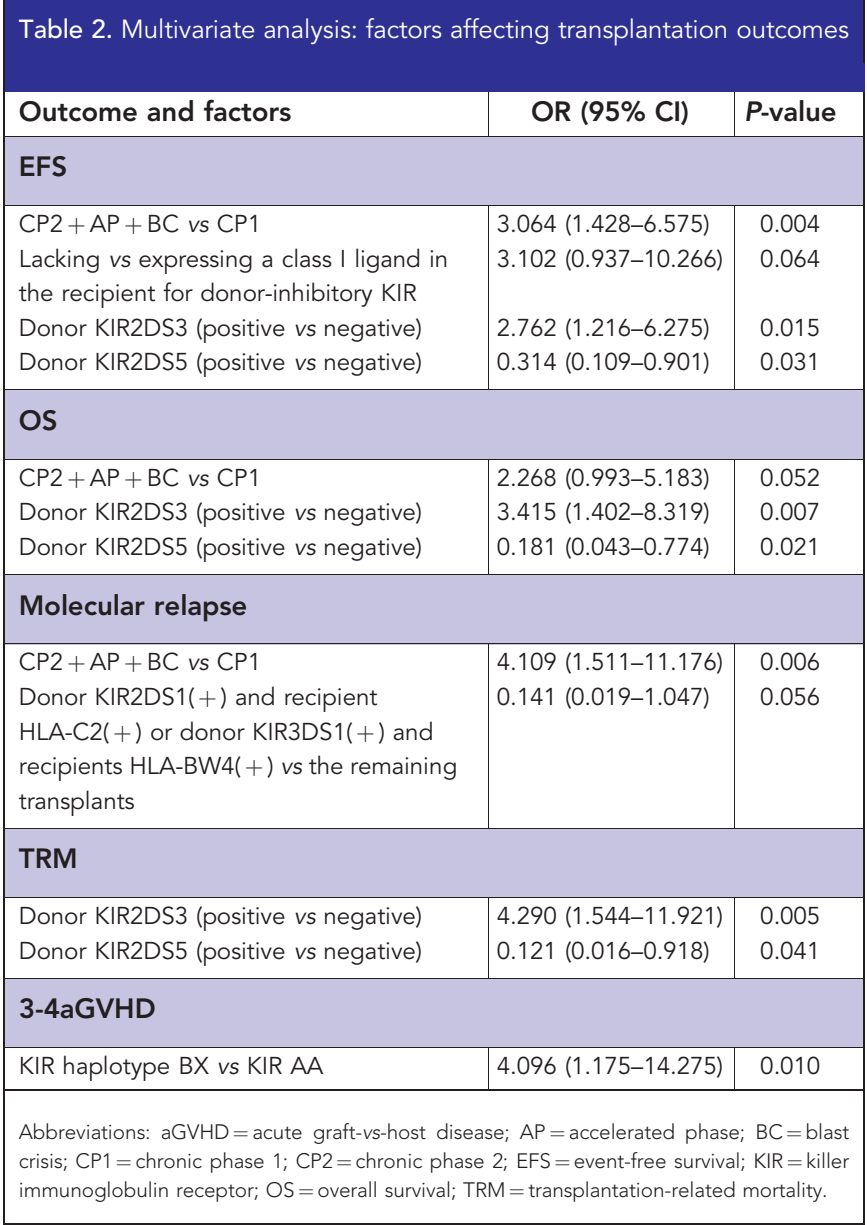

outcomes. The reasons for these apparently discordant results are currently unclear. Further laboratory studies, such as NK-cell functional reconstitution differences between patients with and without a KIR ligand for donor-inhibitory KIR, would be more important (Vago et al, 2008; Triplett et al, 2009; Pfeiffer et al, 2010).

Interestingly, the preventative effects on relapse of activated donor KIR genes with the relevant HLA ligand in the recipients were found in the same group. At the same time, the higher relapse rate in recipient lacking a class I ligand for donor-inhibitory KIR was overcome by accepting donors with an activating KIR gene, including KIR2DS1 and KIR3DS1, if the recipients had the relevant ligand, such as HLA-C2 or Bw4. This finding suggested that in the setting of T-cell-replete haploidentical transplantation, the alloreactive NK cells could be best predicted by the donor-activating KIR gene and the relevant KIR ligand in the recipient. This clinical observation was consistent with the recent basic findings that KIR2DS1-positive NK cells could mediate the alloresponses against the C2 HLA-KIR ligand group in vitro (Chewning et al, 2007; Marcenaro et al, 2011, 2013). This finding was also in accordance with the report from Gabriel et al, 2010 after autologous SCT, which found that the interaction between donor KIR3DS1 and HLA-Bw4 in recipients was predictive for increased NK-cell function, as assessed by the control of leukaemic relapse. In alloreactive NK-cell responses, KIR2DS1 expression represents a remarkable advantage because it allows for the efficient killing of C2/C2 or C1/C2 myelomonocytic DCs and T-cell blasts (Sivori et al, 2011; Marcenaro et al, 2013). However, to reduce the risk of relapse among 1277 patients with AML who underwent unrelated matched or single mismatched HSCT, the data published by
Venstrom et al (2012) supported the preferential selection of a KIR2DS1-positive donor if the donor had HLA-C1/C1 or C1/C2 and was not based on HLA-C in the recipient. Pittari et al (2013) also demonstrated NK-cell tolerance of self-specific activating receptor KIR2DS1 in individuals with the cognate HLA-C2 ligand. Therefore, the reason for these apparently discordant results might be the balance between the activating donor KIR gene in NK-cell education/tolerance induction, as well as the initiation of alloreactivity in NK cells post-transplantation. The induction of tolerance in NK cells that express activating receptors through chronic exposure to the ligand was described in mice (Tripathy et al, 2008). However, in the setting of haploidentical transplantation, the induction of tolerance in NK cells that express activating receptors remains unknown.

The contrasting effects of the missing self model between donors and recipients and between the activated donor KIR genes and the relevant KIR ligands in recipients on relapse in haploidentical transplantation precisely suggest that the presence of class I ligands for the donor-activating or donor-inhibitory KIR gene in the recipient might confer some protection against leukaemic relapse in T-cell-replete haploidentical transplantation. Acquisition of NK-cell activity including inflammatory cytokines production and cytotoxicity have a pivotal role in the graft- $v$ s-leukaemia effect after allogeneic haematopoietic cell transplantation. Natural killer cells can only acquire full functionality post-transplantation when the NK cells have been shown to recognise self-MHC class I, a phenomenon referred to as 'licensing' or 'education' (Kim et al, 2005; Yawata et al, 2006, 2008; Yu et al, 2007; Sun et al, 2009; Sungur et al, 2013). The interaction of recipients class I ligands and donor-inhibitory KIR genes and activating KIR genes have important roles on the process of NK-cell education in vivo and the initiation of alloreactivity in NK cells post-haploidentical transplantation. Therefore, we hypothesis that KIR-HLA interactions and of how NK-cell education undergoes T-cell-replete haploidentical transplantation to dictate NK function would be helpful in revealing these apparently disparate results in the literature in the future. It would also be helpful to explain the contrasting effects of donor KIR2DS5 and donor KIR2DS3 on TRM and survival. However, this study was limited by not performing the NK functional recovery kinetics to explore the correlations between the educated/uneducated NK cells' reconstitution and the alloreactive NK cells' recovery in vivo post-T-cell-replete haploidentical transplantation.

In summary, the seemly contrasting effects of alloreactive NK cells on relapse, using the missing self model or activated donor KIR genes, reflect that the presence of class I ligand in the recipient for the donor-inhibitory KIR gene or the donoractivating KIR gene could be an superior prognostic factor in preventing relapse. This study indicated that the presence of recipient class I ligands for donor-activating and donorinhibitory KIR genes would promote NK-cell functional recovery during NK-cell education, thus conferring some protection against leukaemic relapse in T-cell-repleted, haploidentical transplantation, which would be worthy of future study. In addition, a large diverse cohort study should be conducted in the future in a prospective manner to confirm the effects of recipient HLA and donor KIR genotype associations on the clinical outcomes of transplantation.

\section{ACKNOWLEDGEMENTS}

This work was supported by the National Natural Science Foundation of China (grant nos. 81270644 and 81230013), the Major State Basic Research Development Program of China 
(973 Program No. 2013CB733700), and doctoral funding from the Ministry of Education of China (grant no. 20110001110039), and partially supported by Collaborative Innovation Center of Hematology, China. We thank all of the core facilities at the Peking University Institute of Hematology for sample collection.

\section{CONFLICT OF INTEREST}

The authors declare no conflict of interest.

\section{REFERENCES}

Chewning JH, Gudme CN, Hsu KC, Selvakumar A, Dupont B (2007) KIR2DS1-positive NK cells mediate alloresponse against the C2 HLA-KIR ligand group in vitro. J Immunol 179: 854-868.

Fuchs EJ, Huang XJ, Miller JS (2010) HLA-haploidentical stem cell transplantation for hematologic malignancies. Biol Blood Marrow Transplant 16: S57-S63.

Gabriel IH, Sergeant R, Szydlo R, Apperley JF, deLavallade H, Alsuliman A, Khoder A, Marin D, Kanfer E, Cooper N, Davis J, MacDonald D, Bua M, Foroni L, Giles C, Milojkovic D, Rahemtulla A, Rezvani K (2010) Interaction between KIR3DS1 and HLA-Bw4 predicts for progression-free survival after autologous stem cell transplantation in patients with multiple myeloma. Blood 116: 2033-2039.

Huang XJ (2008) Current status of haploidentical stem cell transplantation for leukemia. J Hematol Oncol 1: 27.

Huang XJ, Zhao X.Y, Liu DH, Liu KY, Xu LP (2007) Deleterious effects of KIR ligand incompatibility on clinical outcomes in haploidentical hematopoietic stem cell transplantation without in vitro T-cell depletion. Leukemia 21: 848-851.

Jiang Q, Xu LP, Liu DH, Liu KY, Chen SS, Jiang B, Jiang H, Chen H, Chen YH, Han W, Zhang XH, Wang Y, Qin YZ, Liu YR, Lai YY, Huang XJ (2011) Imatinib mesylate versus allogeneic hematopoietic stem cell transplantation for patients with chronic myelogenous leukemia in the accelerated phase. Blood 117: 3032-3040.

Kijima M, Gardiol N, Held W (2011) Natural killer cell mediated missing-self recognition can protect mice from primary chronic myeloid leukemia in vivo. PLoS One 6: e27639.

Kim S, Poursine-Laurent J, Truscott SM, Lybarger L, Song YJ, Yang L, qFrench AR, Sunwoo JB, Lemieux S, Hansen TH, Yokoyama WM (2005) Licensing of natural killer cells by host major histocompatibility complex class I molecules. Nature 436: 709-713.

Leung W, Iyengar R, Triplett B, Turner V, Behm FG, Holladay MS, Houston J, Handgretinger R (2005) Comparison of killer Ig-like receptor genotyping and phenotyping for selection of allogeneic blood stem cell donors. J Immunol 174: 6540-6545.

Leung W, Iyengar R, Turner V, Lang P, Bader P, Conn P, Niethammer D, Handgretinger R (2004) Determinants of antileukemia effects of allogeneic NK cells. J Immunol 172: 644-650.

Marcenaro E, Carlomagno S, Pesce S, Della Chiesa M, Moretta A, Sivori S (2011) Role of alloreactive KIR2DS1(+) NK cells in haploidentical hematopoietic stem cell transplantation. J Leukocyte Biol 90: 661-667.

Marcenaro E, Pesce S, Sivori S, Carlomagno S, Moretta L, Moretta A (2013) KIR2DS1-dependent acquisition of CCR7 and migratory properties by human NK cells interacting with allogeneic HLA-C2 + DC or T cell blasts. Blood 121: 3396-3401.

Pfeiffer MM, Feuchtinger T, Teltschik HM, Schumm M, Muller I, Handgretinger R, Lang P (2010) Reconstitution of natural killer cell receptors influences natural killer activity and relapse rate after haploidentical transplantation of T- and B-cell depleted grafts in children. Haematologica 95: 1381-1388.

Pittari G, Liu X-R, Selvakumar A, Zhao Z, Merino E, Huse M, Chewning JH, Hsu KC, Dupont B (2013) NK cell tolerance of self-specific activating receptor KIR2DS1 in individuals with cognate HLA-C2 ligand. J Immunol 190: $4650-4660$

Raulet DH, Vance RE (2006) Self-tolerance of natural killer cells. Nat Rev Immunol 6: 520-531.

Ruggeri L, Capanni M, Casucci M, Volpi I, Tosti A, Perruccio K, Urbani E, Negrin RS, Martelli MF, Velardi A (1999) Role of natural killer cell alloreactivity in HLA-mismatched hematopoietic stem cell transplantation. Blood 94: 333-339.

Ruggeri L, Capanni M, Urbani E, Perruccio K, Shlomchik WD, Tosti A, Posati S, Rogaia D, Frassoni F, Aversa F, Martelli MF, Velardi A (2002) Effectiveness of donor natural killer cell alloreactivity in mismatched hematopoietic transplants. Science 295: 2097-2100.

Ruggeri L, Mancusi A, Capanni M, Urbani E, Carotti A, Aloisi T, Stern M, Pende D, Perruccio K, Burchielli E, Topini F, Bianchi E, Aversa F, Martelli MF, Velardi A (2007) Donor natural killer cell allorecognition of missing self in haploidentical hematopoietic transplantation for acute myeloid leukemia: challenging its predictive value. Blood 110: 433-440.

Simula MP, Marktel S, Fozza C, Kaeda J, Szydlo RM, Nadal E, Bua M, Rahemtulla A, Kanfer E, Marin D, Olavarria E, Goldman JM, Apperley JF, Dazzi F (2007) Response to donor lymphocyte infusions for chronic myeloid leukemia is dose-dependent: the importance of escalating the cell dose to maximize therapeutic efficacy. Leukemia 21: 943-948.

Sivori S, Carlomagno S, Falco M, Romeo E, Moretta L, Moretta A (2011) Natural killer cells expressing the KIR2DS1-activating receptor efficiently kill T-cell blasts and dendritic cells: implications in haploidentical HSCT. Blood 117: 4284-4292.

Sun JC, Beilke JN, Lanier LL (2009) Adaptive immune features of natural killer cells. Nature 457: 557-561.

Sungur CM, Tang-Feldman YJ, Zamora AE, Alvarez M, Pomeroy C, Murphy WJ (2013) Murine NK-cell licensing is reflective of donor MHC-I following allogeneic hematopoietic stem cell transplantation in murine cytomegalovirus responses. Blood 122: 1518-1521.

Symons HJ, Leffell MS, Rossiter ND, Zahurak M, Jones RJ, Fuchs EJ (2010) Improved survival with inhibitory killer immunoglobulin receptor (KIR) gene mismatches and KIR haplotype B donors after nonmyeloablative, HLA-haploidentical bone marrow transplantation. Biol Blood Marrow Transplant 16: 533-542.

Tripathy SK, Keyel PA, Yang L, Pingel JT, Cheng TP, Schneeberger A, Yokoyama WM (2008) Continuous engagement of a self-specific activation receptor induces NK cell tolerance. J Exp Med 205: 1829-1841.

Triplett BM, Horwitz EM, Iyengar R, Turner V, Holladay MS, Gan K, Behm FG, Leung W (2009) Effects of activating NK cell receptor expression and NK cell reconstitution on the outcomes of unrelated donor hematopoietic cell transplantation for hematologic malignancies. Leukemia 23: 1278-1287.

Vago L, Forno B, Sormani MP, Crocchiolo R, Zino E, Di Terlizzi S, Lupo Stanghellini MT, Mazzi B, Perna SK, Bondanza A, Middleton D, Palini A, Bernardi M, Bacchetta R, Peccatori J, Rossini S, Roncarolo MG, Bordignon C, Bonini C, Ciceri F, Fleischhauer K (2008) Temporal, quantitative, and functional characteristics of single-KIR-positive alloreactive natural killer cell recovery account for impaired graft-versus-leukemia activity after haploidentical hematopoietic stem cell transplantation. Blood 112: 3488-3499.

Venstrom JM, Pittari G, Gooley TA, Chewning JH, Spellman S, Haagenson M, Gallagher MM, Malkki M, Petersdorf E, Dupont B, Hsu KC (2012) HLA-C-dependent prevention of leukemia relapse by donor activating KIR2DS1. N Engl J Med 367: 805-816.

Xiao-jun H, Lan-ping X, Kai-yan L, Dai-hong L, Huan C, Wei H, Yu-hong C, Jing-zhi W, Yao C, Xiao-hui Z, Hong-Xia S, Dao-Pei L (2008) HLAmismatched/haploidentical hematopoietic stem cell transplantation withoutin vitroT cell depletion for chronic myeloid leukemia: improved outcomes in patients in accelerated phase and blast crisis phase. Ann Med 40: 444-455.

Yawata M, Yawata N, Draghi M, Little AM, Partheniou F, Parham P (2006) Roles for HLA and KIR polymorphisms in natural killer cell repertoire selection and modulation of effector function. J Exp Med 203: 633-645.

Yawata M, Yawata N, Draghi M, Partheniou F, Little AM, Parham P (2008) MHC class I-specific inhibitory receptors and their ligands structure diverse human NK-cell repertoires toward a balance of missing selfresponse. Blood 112: 2369-2380.

Yong AS, Keyvanfar K, Hensel N, Eniafe R, Savani BN, Berg M, Lundqvist A, Adams S, Sloand EM, Goldman JM, Childs R, Barrett AJ (2009) Primitive quiescent $\mathrm{CD} 34+$ cells in chronic myeloid leukemia are targeted by in vitro expanded natural killer cells, which are functionally enhanced by bortezomib. Blood 113: 875-882.

Yu J, Heller G, Chewning J, Kim S, Yokoyama W.M, Hsu KC (2007) Hierarchy of the human natural killer cell response is determined by class 
and quantity of inhibitory receptors for self-HLA-B and HLA-C ligands. J Immunol 179: 5977-5989.

Zhao XY, Chang YJ, Huang XJ (2011) Conflicting impact of alloreactive NK cells on transplantation outcomes after haploidentical transplantation: do the reconstitution kinetics of natural killer cells create these differences? Biol Blood Marrow Transplant 17: 1436-1442.

Zhao XY, Huang XJ, Liu KY, Xu LP, Liu DH (2007) Prognosis after unmanipulated HLA-haploidentical blood and marrow transplantation is correlated to the numbers of KIR ligands in recipients. Eur J Haematol 78: $338-346$.

This work is published under the standard license to publish agreement. After 12 months the work will become freely available and the license terms will switch to a Creative Commons AttributionNonCommercial-Share Alike 3.0 Unported License. 